\title{
On the units of bipartite entanglement: Is sixteen ounces of entanglement always equal to one pound?
}

\author{
M. A. Nielsen \\ Center for Quantum Computer Technology, University of Queensland 4072, Australia
}

(October 29, 2018)

\begin{abstract}
In a good physical theory dimensionless quantities such as the ratio $m_{p} / m_{e}$ of the mass of the proton to the mass of the electron do not depend on the system of units being used. This paper demonstrates that one widely used method for defining measures of entanglement violates this principle. Specifically, in this approach dimensionless ratios $E(\rho) / E(\sigma)$ of entanglement measures may depend on what state is chosen as the basic unit of entanglement. This observation leads us to suggest three novel approaches to the quantification of entanglement. These approaches lead to unit-free definitions for the entanglement of formation and the distillable entanglement, and suggest natural measures of entanglement for multipartite systems. We also show that the behaviour of one of these novel measures, the entanglement of computation, is related to some open problems in computational complexity.
\end{abstract}

PACS Numbers: 03.65.Bz, 03.67.-a

\section{INTRODUCTION}

Quantum mechanics harbours a rich structure whose investigation and explication is the goal of quantum information science [1]:2]. Of particular interest is the development of a quantitative theory of entangled quantum states. This theory is still a work in progress; see for example [3 12] for a sample of the ongoing work, and further references.

The present paper is a two-pronged contribution to the theory of entanglement. The first goal of the paper, explored in detail in Section [1], is to deliver a critique of a commonly used approach to the definition of measures of entanglement, identifying what we refer to as a ratio problem arising in this approach. This ratio problem manifests itself in, for example, definitions for measures of entanglement such as the entanglement of formation and distillable entanglement. The second goal of the paper, explored in detail in Section III, is to develop approaches to the quantification of entanglement that do not suffer the ratio problem. In particular, we describe three novel approaches to the quantification of entanglement without the ratio problem arising. These new approaches lead to methods for defining the entanglement of formation and distillable entanglement that don't suffer the ratio problem, and also lead to interesting connections between entanglement measures and computational complexity. Section IV] concludes the paper.

Before proceeding to the detailed discussion in Sections II through IV, it is useful to take a broad look at how the ratio problem arises, and to consider some of the issues inherent in attempts to critique definitions of entanglement. A central notion in much existing work on the quantification of entanglement is the concept of a "standard unit" of entanglement, usually chosen to be the Bell state of two qubits, $(|00\rangle+|11\rangle) / \sqrt{2}$. Naturally, one may ask whether the quantitative theory of entanglement changes in any essential way when the standard unit of entanglement is changed to be a state other than the Bell state? In general, in a good physical theory dimensionless quantities such as the ratio $m_{p} / m_{e}$ of the mass of the proton to the mass of the electron should not depend on the system of units being used, whether they be kilograms, Planck masses, or any other unit. For convenience, I will refer to this unit-invariance as the ratio property. In this paper we show that the usual method of defining the entanglement of formation and the distillable entanglements don't satisfy the ratio property: for both these measures, ratios $E(\rho) / E(\sigma)$ of the entanglement present in states $\rho$ and $\sigma$ depend on what standard unit of entanglement is chosen, in contradiction with our expectations for well-behaved physical quantities. This is the ratio problem.

How important is the ratio problem to ongoing attempts to construct a theory of entanglement? As explained in more detail below, I believe considerable caution is warranted in assessing criticisms of measures of entanglement which are of an axiomatic nature, as is the ratio problem. In particular, I don't believe that the ratio problem alone is sufficient to invalidate the notion of defining measures of entanglement based on a standard unit of entanglement; I think that this notion can and should be further developed, as is being done in, for example, [13 15]. On the other hand, I believe that the ratio problem is of sufficient importance that it should strongly motivate the search for other approaches to defining measures of entanglement which don't suffer this problem, and some progress along these lines is reported later in this paper.

I commented above that caution is necessary in assessing criticisms of an axiomatic nature. The reason for 
this comment is the general poverty of such axiomatic approaches. By this, I mean that although there is a tendency when defining measures of entanglement to focus on "nice" elementary properties one would like such a measure to have (such as the ratio property), what we really want is deep theorems connecting measures of entanglement in surprising ways to other problems in quantum information science. An analogous situation occurred in the early days of information theory: the Shannon entropy derives its goodness as a measure of information not from its nice elementary properties, as described in any information theory text, but from deep theorems connecting the entropy to a priori unrelated problems of data compression, communication in the presence of noise, and so on. At the present time I believe it is fair to say that few deep results connecting measures of entanglement to other problems are known. Of course, in the absence of such deep results, critiques (in particular, this paper) of existing definitions of entanglement are necessarily of an axiomatic nature, but the above caveats need to be borne in mind when assessing such critiques.

\section{THE RATIO PROBLEM}

The entanglement of formation and the distillable entanglement are two of the best known measures of entanglement for bipartite quantum systems [3]. We now review the standard definitions of these measures, based on the Bell state as the standard unit of entanglement, writing the entanglement of formation for a general quantum state $\rho$ as $F(\rho)$, and the distillable entanglement as $D(\rho)$. In this approach the entanglement of formation is defined as follows. Imagine Alice and Bob have a protocol that, given $m$ shared Bell states, allows them to create $n$ "pretty good" copies of $\rho$, using only local operations on their respective systems, and classical communication - a so-called "LOCC" protocol. These copies of $\rho$ must be pretty good in the sense that the fidelity between $\rho^{\otimes n}$ and the state created by the protocol tends to 1 as $m$ and $n$ tend to infinity. The entanglement of formation is defined to be the minimum value that may be achieved for the ratio $m / n$, in the limit as $n$ becomes large, where the minimum is performed over all possible LOCC protocols. Schematically, we have:

$$
n F(\rho) \times \text { Bell } \rightarrow n \times \rho,
$$

where the $\rightarrow$ indicates a LOCC protocol. Thus, the entanglement of formation is a measure of how cheaply $\rho$ may be created, given Bell state entanglement and the ability to perform local operations and classical communication.

In this Bell-state-as-standard-unit approach the distillable entanglement is defined in a similar asymptotic way. Specifically, consider a LOCC protocol in which Alice and Bob start out sharing $n$ copies of $\rho$, and produce $m$ good copies of the Bell state. The distillable entanglement is defined as the maximum value that may be achieved for the ratio $m / n$, in the limit as $n$ becomes large. Schematically, we have:

$$
n \times \rho \rightarrow n D(\rho) \times \text { Bell } .
$$

Thus, the distillable entanglement is a measure of the Bell state entanglement yield which may be produced if Alice and Bob start with copies of $\rho$ and are given the ability to perform local operations and classical communication.

An impressive but incomplete body of knowledge exists about the entanglement of formation and distillable entanglement. For pure states $|\psi\rangle$ both quantities are equal to the entropy of Alice's reduced density matrix [16, 3], $\rho_{\psi} \equiv \operatorname{tr}_{B}(|\psi\rangle\langle\psi|)$, that is, $F(|\psi\rangle\langle\psi|)=D(|\psi\rangle\langle\psi|)=$ $S\left(\rho_{\psi}\right)$. For the case of mixed states of two qubits, Wootters [17] has provided an elegant formula for the entanglement of formation, subject to the proof of the additivity conjecture for entanglement of formation. (This conjecture has not yet been proved, but is widely believed to be true after numerical testing.) The distillable entanglement has been evaluated or bounded for a variety of classes of mixed states (for a selection of work and further references see 18 21. (6]), however no general formula is known, even for the case of a mixed state of two qubits.

Suppose we change the standard unit of entanglement so it is no longer the Bell state, defining a new "entanglement of formation" and "distillable entanglement" with respect to a new standard unit of entanglement, the state $\sigma$. We denote these new concepts as $F_{\sigma}(\rho)$ and $D_{\sigma}(\rho)$, and refer to them as the $\sigma$-entanglement of formation, and the $\sigma$-distillable entanglement. More precisely, the $\sigma$-entanglement of formation is defined as the minimal possible value that may be achieved for the ratio $m / n$, in the limit as $n$ becomes large, for a LOCC protocol in which Alice and Bob initially share $m$ copies of $\sigma$, and produce $n$ pretty good copies of $\rho$, with the same asymptotic fidelity requirements as before. The $\sigma$-distillable entanglement is defined as the maximal possible value that may be achieved for the ratio $m / n$, in the limit as $n$ becomes large, for a LOCC protocol in which Alice and Bob initially share $n$ copies of $\rho$, and produce $m$ pretty good copies of $\sigma$.

With these definitions it is easy to prove the inequalities

$$
\begin{gathered}
D(\rho) \leq F_{\sigma}(\rho) D(\sigma) \leq F(\rho) \leq F_{\sigma}(\rho) F(\sigma) \\
D_{\sigma}(\rho) D(\sigma) \leq D(\rho) \leq D_{\sigma}(\rho) F(\sigma) \leq F(\rho) .
\end{gathered}
$$

We prove the inequalities in (14); the inequalities in (3) follow by similar reasoning. In general it will be convenient to concentrate the discussion on $D_{\sigma}(\rho)$, since the properties of $F_{\sigma}(\rho)$ are similar. By definition of 
the distillable entanglement and entanglement of formation, there exist asymptotically good LOCC protocols achieving the transformations $n \times \rho \rightarrow n D(\rho) \times$ Bell and $n D(\rho) \times$ Bell $\rightarrow n D(\rho) / F(\sigma) \times \sigma$, and thus there is an asymptotically good protocol achieving the transformation $n \times \rho \rightarrow n D(\rho) / F(\sigma) \times \sigma$. By definition of the $\sigma$-distillable entanglement, it follows that $D_{\sigma}(\rho) \geq$ $D(\rho) / F(\sigma)$, which is the second inequality in (4). To prove the third inequality, note that by definition of the $\sigma$-distillable entanglement there exists an asymptotically good LOCC protocol $n \times \rho \rightarrow n D_{\sigma}(\rho) \times \sigma$. By definition of the entanglement of formation, there exists an asymptotically good LOCC protocol $n F(\rho) \times$ Bell $\rightarrow n \times \rho$, and thus there exists an asymptotically good LOCC protocol $n F(\rho) \times$ Bell $\rightarrow n D_{\sigma}(\rho)$, from which it follows that $F(\sigma) \leq F(\rho) / D_{\sigma}(\rho)$, which is the third inequality in (4). The first inequality follows by noting that there are asymptotically good LOCC protocols $n \times \rho \rightarrow n D_{\sigma}(\rho) \times \sigma \rightarrow n D_{\sigma}(\rho) D(\sigma) \times$ Bell, and thus $D(\rho) \geq D_{\sigma}(\rho) D(\sigma)$.

Suppose $\sigma$ is a state whose distillable entanglement is strictly less than the entanglement of formation, $D(\sigma)<$ $F(\sigma)$. Such states have been proved to exist in [22]. Intuitively it is clear that $D_{\sigma}(\sigma)=1$, and we prove this rigorously below. From the definition it follows immediately that $D_{\sigma}($ Bell $)=1 / F(\sigma)$, and thus

$$
\frac{D_{\sigma}(\sigma)}{D_{\sigma}(\text { Bell })}=F(\sigma)>D(\sigma)=\frac{D(\sigma)}{D(\text { Bell })}
$$

providing an example where dimensionless ratios of entanglement quantities depend upon the unit of entanglement being used. To conclude, we need only show that $D_{\sigma}(\sigma)=1$. Clearly, $D_{\sigma}(\sigma) \geq 1$; we need only worry that it might be possible for Alice and Bob to distill more than one copy of $\sigma$ per copy of $\sigma$ that they are given. (Note that this is possible when $\sigma$ is separable, since additional copies of $\sigma$ can be prepared by LOCC.) For a contradiction, suppose that $D_{\sigma}(\sigma)>m / n>1$, where $m$ and $n$ are large positive integers. Then in the asymptotic limit of large $m$ and $n$, we are able to accomplish the transformation $\sigma \times n \rightarrow \sigma \times m$ with high fidelity. Assuming the additivity conjecture for entanglement of formation, and using the continuity results of Nielsen [23] and the fact that the entanglement of formation is non-increasing under LOCC, we obtain $n F(\sigma) \geq m F(\sigma)$, that is, $n \geq m$, the desired contradiction.

Similar results are easily obtained for the $\sigma$ entanglement of formation. Specifically, suppose $D(\sigma)<$ $F(\sigma)$. It is easily verified that $F_{\sigma}(\sigma)=1$ and $F_{\sigma}($ Bell $)=$ $1 / D(\sigma)$, so

$$
\frac{F_{\sigma}(\sigma)}{F_{\sigma}(\text { Bell })}=D(\sigma)<F(\sigma)=\frac{F(\sigma)}{F(\text { Bell })},
$$

showing that the ratio problem arises for both the distillable entanglement and the entanglement of formation.
We have shown that the usual definitions for the entanglement of formation and distillable entanglement do not satisfy the ratio property. In the introduction some remarks were made about how seriously such a problem needs to be taken. Further to those remarks, the ratio problem could possibly be disregarded on the grounds that it is "unnatural" to choose a mixed state as the standard unit of entanglement, or that the Bell state really is, in some sense, special. I don't believe that such a position is valid if one takes seriously the notion that quantifying the amount of entanglement present in a mixed state is a sensible objective; surely if this is the case then it makes sense to change the unit of entanglement which one uses, and expect dimensionless ratios to remain invariant under such changes in the unit. To continue the analogy with mass: while there are serious practical concerns of stability, repeatability and ease-of-duplication when using poorly chosen standards of mass (say, one mass unit $\equiv$ one Indian elephant), from an ideal theoretical viewpoint this is just as satisfactory as using, say, electrons as the mass standard.

\section{NOVEL APPROACHES TO THE DEFINITION OF ENTANGLEMENT}

The ratio problem suggests that it might be useful to develop approaches to quantifying entanglement which do not suffer this problem. Some such approaches already been proposed in the existing literature, such as the relative entropy of entanglement [6], which avoids the ratio problem by not singling out any special standard unit of entanglement - they are "unit-free". We now describe several new methods for defining entanglement which do not suffer the ratio problem. Furthermore, we will show that these definitions give rise to unit-free definitions of the entanglement of formation and distillable entanglement, definitions which do not suffer the ratio problem! What then have we gained by switching to new definitions? The answer is two-fold. First, from a fundamental point of view, definitions of entanglement which do not satisfy the ratio problem are a priori more satisfactory. Second, these alternative definitions generalize in a natural way to multipartite systems, and we will see that they have natural connections to problems in quantum computational complexity, quantum communication, and distributed quantum computation.

Our first alternative approach to the definition of an entanglement measure is the entanglement of creation, $E_{\mathrm{cr}}(\rho)$, defined as follows. Alice and Bob wish to create some large number $n$ of good copies of the state $\rho$. They start with no preshared entanglement, but can use qubits to communicate (at a cost), and can do classical communication for free. The entanglement of creation is defined to be the asymptotically minimal value for the ratio of the number of qubits $m$ they transmit to the number of 
copies $n$ of $\rho$ they create, with the requirement that as $n$ becomes large the copies of $\rho$ created must be good copies. This definition is obviously independent of any concept of a standard unit of entanglement, and thus can not possibly suffer from the ratio problem. Note that we do use a specific "standard" quantum channel for Alice and Bob to communicate - a noiseless quantum channel - however it seems clear that this channel really is special among all possible communication channels.

With this definition, the entanglement of creation is equal to the entanglement of formation, by the following argument. Suppose we are given a protocol for creating $n$ copies of $\rho$ using $n E_{\mathrm{cr}}(\rho)$ qubits of communication between Alice and Bob, plus classical communication. Then this is easily simulated using $n E_{\mathrm{cr}}(\rho)$ shared Bell states, classical communication, and no quantum communication, using quantum teleportation [24]. Thus, $F(\rho) \leq E_{\text {cr }}(\rho)$. Conversely, suppose we are given a protocol to create $n$ copies of $\rho$ using $n F(\rho)$ shared Bell states, local operations and classical communication. This may be simulated using $n F(\rho)$ qubits of communication to set up the $n F(\rho)$ shared Bell states, and classical communication. Thus, $E_{\text {cr }}(\rho) \leq F(\rho)$, from which we deduce that $E_{\text {cr }}(\rho)=F(\rho)$, as claimed.

Our second alternative approach to the definition of entanglement is the entanglement of communication. Suppose Alice and Bob share a large number $n$ of copies of a state $\rho$. The entanglement of communication $E_{\text {comm }}(\rho)$ of $\rho$ is defined to be the asymptotically maximal value for the ratio $m / n$ of the number of qubits $m$ Alice can transmit to Bob with asymptotically high fidelity, using LOCC, and $n$ preshared copies of $\rho$. With this definition the entanglement of communication is exactly equal to the distillable entanglement, as may be seen by the following argument. By definition, there exists a LOCC protocol for Alice and Bob to convert $n$ copies of $\rho$ into $n D(\rho)$ copies of the Bell state. Then using teleportation 24 and additional classical communication Alice can transmit $n D(\rho)$ qubits to Bob. Thus $D(\rho) \leq E_{\text {comm }}(\rho)$. To show the reverse inequality, suppose Alice and Bob share $n$ copies of $\rho$. Alice creates $n E_{\text {comm }}(\rho)$ Bell states locally, and then transmits half of each Bell state to Bob, creating $n E_{\text {comm }}(\rho)$ shared Bell states, and establishing that $E_{\text {comm }}(\rho) \leq D(\rho)$, from which we deduce that $E_{\text {comm }}(\rho)=D(\rho)$, as claimed. As for the entanglement of creation, the entanglement of communication is obviously independent of any concept of a standard unit of entanglement, and thus can not possibly suffer from the ratio problem.

As we have shown, the entanglement of creation and entanglement of communication are not truly new measures of entanglement. Rather, they offer unit-free methods for defining the quantity of entanglement present in a system. A significant advantage of both methods of definition over the standard approach is that they suggest natural generalizations to multipartite systems, where it is not at all clear what the appropriate standard unit of entanglement is. For example, for an $n$ party system, it is possible to define the entanglement of creation for a state $\rho$ to be the minimal asymptotic amount of qubit communication between the parties, per good copy of the state created. In the case of the entanglement of communication, making appropriate definitions is a little more tricky; however, as the following example illustrates, there are some natural starting points. One possible way to go is to define the entanglement of communication for a state $\rho$ as the total number of qubits of communication which can be done (between all possible pairs of parties), per copy of $\rho$. Other natural variants of this measure also suggest themselves, perhaps counting the number of qubits of communication that can be achieved between some specified pair of parties, or some other natural communication goal. These measures of entanglement will be investigated in more detail elsewhere, however for now the main point is that they provide illustrative examples of alternative approaches to the definition of the entanglement of formation and distillable entanglement which do not suffer the ratio problem, and yield useful generalizations.

Our third and final alternative approach to the quantification of entanglement is the entanglement of computation, which is best illustrated via an example. Suppose we set $U$ to be the controlled phase gate [1] on two qubits, that is, the gate which takes $|x, y\rangle$ to $(-1)^{x y}|x, y\rangle$. The idea is that Alice and Bob are able (at a cost) to apply the gate $U$ jointly to one of Alice's qubits and to one of Bob's qubits. We also allow them to communicate classically for free; what happens when free classical communication is not allowed will be investigated elsewhere. The entanglement of computation for $\rho$ with respect to $U, E_{U}(\rho)$, is defined to be the minimal asympotic value for the ratio $m / n$ of the number of times $m$ the gate $U$ must be applied to create $n$ pretty good copies of the state $\rho$. Obviously, there is no standard unit of entanglement invoked in this definition, and in this sense the entanglement of computation does not suffer the ratio problem. Of course, we did single out a standard gate, $U$, in our definition, and we will ask below whether this might cause some analogue of the ratio problem to arise for the entanglement of computation. In the specific case where $U$ is the controlled phase gate, it is easily seen that $\left.E_{U}(\rho)=F(\rho)\right)$. This follows from the results of [25], where it was shown that, given the ability to communicate classically, one shared Bell state is equivalent to the ability to perform a controlled-NOT (or equivalently, a controlled phase) gate.

We have seen that for a specific choice of gate $U$ the entanglement of computation is equal to the entanglement of formation, however in general it is not obvious that this is the case. Indeed, it is conceivable that ratios like $E_{U}(\rho) / E_{U}(\sigma)$ may depend on the quantum gate $U$ chosen as the standard unit, creating a sort of ratio problem for the entanglement of computation. I conjec- 
ture that such a situation does in fact occur, but have not yet proved it; this issue will be explored in more detail elsewhere. Furthermore, it is not obvious that the definition is symmetric under interchange of the role of Alice and Bob; this is true in the case when $U$ is chosen to be the controlled phase gate, however for gates without the symmetry of the controlled phase gate it is not so obvious.

Despite these possible difficulties a major advantage of the entanglement of computation is that it generalizes to multipartite systems in an obvious way. For example, for a system consisting of $n$ qubits, we can define the $n$ party entanglement of computation for a state $\rho$, with respect to the controlled phase gate, to be the number of controlled phase gates required, asymptotically, to produce a copy of $\rho$, with local operations on individual qubits and classical communication allowed. It follows from results of Knill [26] that for most states of $n$ qubits this measure of entanglement is, in general, exponential in the number of qubits $n$ in the system.

This generalization to $n$ qubits allows us to deduce an interesting connection between the entanglement of computation and computational complexity theory, using a method similar to that developed in Chapter 6 of [27, where entanglement measures were used as a tool to deduce bounds on distributed quantum computation. Suppose we consider the state

$$
\left|\psi_{n}\right\rangle \equiv \sum_{G}|G\rangle|f(G)\rangle,
$$

where the sum is over all graphs $G$ on $n$ vertices, $|G\rangle$ is some appropriate representation for $G$ in the computational basis, such as a listing of the elements of the adjacency matrix for $G$, and $f(G) \in\{0,1\}$ is some binary function of $G$. For example, we might define $f(G) \equiv 0$ if $G$ has no Hamiltonian cycle, while $f(G) \equiv 1$ if $G$ has a Hamiltonian cycle. Suppose we have a quantum algorithm to compute $f(G)$ in time $g(n)$. It follows that the entanglement of computation for $\left|\psi_{n}\right\rangle$ must scale as $O(g(n))$, with some small overhead necessary to prepare the superposition $\sum_{G}|G\rangle$. Suppose it were possible to prove that for this choice of function $f$, the entanglement of computation for the state $\left|\psi_{n}\right\rangle$ behaved in a manner superpolynomial in $n^{1}$. Then it would follows that QP, the class of languages accepted with probability one on a quantum Turing machine operating in polynomial time, is not equal to the class NP of languages accepted by a classical non-deterministic Turing machine. This would further imply that $\mathbf{P} \neq \mathbf{N P}$, settling one of the great

\footnotetext{
${ }^{1}$ Strictly speaking, an extra system containing working qubits should be appended to the state $\left|\psi_{n}\right\rangle$ to make this line of argument rigorous. Without loss of generality we may assume that the working qubits are in the all $|0\rangle$ state.
}

open problems of theoretical computer science. Thus, the problem of determining the quantity of entanglement present in a quantum state are of relevance to questions about computational complexity theory. (It is interesting to note that similar lines of thought can be explored in relation to the complexity class BQP, although additional continuity properties are required for entanglement measures in this case.) For this line of attack on questions about computational complexity I believe it is quite encouraging that the entanglement of computation can be calculated exactly in the two qubit case [17], and for calculation in the $n$ qubit case our large body of knowledge concerning asymptotic behaviour (especially various laws of large numbers) is encouraging, as is recent progress on classifying the orbits of the $n$ qubit local unitary group (see for example 28 31], and references therein).

A caveat about this approach to problems in computational complexity is in order. Formally, the state $\left|\psi_{n}\right\rangle$ is rather similar to a classical probability distribution on ordered pairs $(G, x)$, where $G$ is an $n$ vertex graph and $x$ a single bit, defined by

$$
p(G, x) \equiv\left\{\begin{array}{l}
1 / M \text { if } x=f(G) \\
0 \text { if } x \neq f(G)
\end{array}\right.
$$

where $M$ is the total number of graphs on $n$ vertices. Once could define a notion of "computational correlation" measuring the amount of correlation in this probability distribution, analogous to the entanglement of computation, except using classical gates and randomness to create the probability distribution. Results about computational complexity would then follow from results about the rate of growth of the computational correlation of the distribution $p$ as $n$ grows, in a fashion exactly analogous to the argument outlined above for the entanglement of computation. This is, perhaps, an approach that could be taken to open problems in computational complexity; nonetheless, I believe that there is more hope in the quantum approach, since quantum information theory has arguably a more elegant structure than classical information theory, as indicated for example by Wootters' beautiful work [17] on the entanglement of formation (and thus entanglement of computation) for two qubits.

\section{CONCLUSION}

The present paper has focused on the axiomatics of entanglement. The problems addressed are elementary, but 
fundamentally influence how one thinks about generalizations of entanglement measures to multipartite systems. In particular, I believe that the approach to entanglement measures based upon the notion of a standard unit of entanglement, while fruitful, needs to be supplemented by other approaches to making such definitions, such as the relative entropy of entanglement, and the three approaches suggested here. At present it is not clear what approach to the quantification of entanglement will ultimately yield the most insight, so it is crucial to think critically about existing approaches, and to develop conceptually novel approaches to the quantification of entanglement.

\section{ACKNOWLEDGMENTS}

Thanks to Carl Caves, Julia Kempe and Damian Pope for stimulating discussions about entanglement.

[1] M. A. Nielsen and I. L. Chuang, Quantum computation and quantum information (Cambridge University Press, Cambridge, 2000).

[2] J. Preskill, Physics 229: Advanced mathematical methods of physics - Quantum computation and information (California Institute of Technology, Pasadena, CA, 1998), http://www.theory.caltech.edu/people/preskill/ph229/.

[3] C. H. Bennett, D. P. DiVincenzo, J. A. Smolin, and W. K. Wootters, Phys. Rev. A 54, 3824 (1996), arXive eprint quant-ph/9604024.

[4] M. A. Nielsen, Phys. Rev. Lett. 83, 436 (1999).

[5] M. Horodecki, P. Horodecki, and R. Horodecki, Phys. Lett. A 223, 1 (1996), arXive eprint quant-ph/9605038.

[6] V. Vedral and M. B. Plenio, Phys. Rev. A 57, 1619 (1998).
[7] G. Vidal, Phys. Rev. Lett. 83, 1046 (1999).

[8] G. Vidal, J. Mod. Opt. 47, 355 (2000).

[9] D. Jonathan and M. B. Plenio, Phys. Rev. Lett. 83, 1455 (1999), arXive eprint quant-ph/9903054.

[10] C. H. Bennett et al., Phys. Rev. Lett. 82, 5385 (1999).

[11] B. M. Terhal, Phys. Lett. A 271, 319 (2000).

[12] W. Dür, J. I. Cirac, M. Lewenstein, and D. Bruß, Phys. Rev. A 61, 2313 (2000).

[13] C. H. Bennett et al., arXive eprint quant-ph/9908073 (1999).

[14] N. Linden, S. Popescu, B. Schumacher, and M. Westmoreland, arXive eprint quant-ph/9912039 (1999).

[15] G. Vidal, W. Dür, and J. I. Cirac, Phys. Rev. Lett. 85, 658 (2000).

[16] C. H. Bennett, H. J. Bernstein, S. Popescu, and B. Schumacher, Phys. Rev. A 53, 2046 (1996), arXive eprint quant-ph/9511030.

[17] W. K. Wootters, Phys. Rev. Lett. 80, 2245 (1998).

[18] E. Rains, arXive eprint quant-ph/0008047 (2000).

[19] P. W. Shor, J. A. Smolin, and B. m. Terhal, arXive eprint quant-ph/0010054 (2000).

[20] J. Eisert et al., Phys. Rev. Lett. 84, 1611 (2000), arXive eprint quant-ph/9907021.

[21] E. M. Rains, IEEE Trans. Inf. Theory 44, 1388 (1998).

[22] M. Horodecki, P. Horodecki, and R. Horodecki, Phys. Rev. Lett. 84, 4260 (2000), arXive eprint quant$\mathrm{ph} / 9912076$.

[23] M. A. Nielsen, Phys. Rev. A 61, 04301 (2000), arXive eprint quant-ph/9908086.

[24] C. H. Bennett et al., Phys. Rev. Lett. 70, 1895 (1993).

[25] D. Collins, N. Linden, and S. Popescu, arXive eprint quant-ph/0005102 (2000).

[26] E. Knill, arXive eprint quant-ph/9508006 (1995).

[27] M. A. Nielsen, Ph.D. thesis, University of New Mexico, 1998, arXive eprint quant-ph/0011036.

[28] H. Carteret, A. Higuchi, and A. Sudbery, arXive eprint quant-ph/0006125 (2000).

[29] A. Acin et al., Phys. Rev. Lett. 85, 1560 (2000).

[30] N. Linden and S. Popescu, Fortsch. Phys. 46, 567 (1998), arXive eprint quant-ph/9711016.

[31] M. Grassl, M. Roetteler, and T. Beth, Phys. Rev. A 58, 1833 (1998), arXive eprint quant-ph/9712040. 